\title{
Oscillation mode linewidths and heights of 23 main-sequence stars observed by Kepler (Corrigendum)
}

\author{
T. Appourchaux ${ }^{1}$, H. M. Antia ${ }^{2}$, O. Benomar ${ }^{3,4}$, T. L. Campante ${ }^{5,9}$, G. R. Davies ${ }^{5,10}$, R. Handberg ${ }^{5,9}$, R. Howe , $^{5}$ \\ C. Régulo ${ }^{6,7}$, K. Belkacem ${ }^{8}$, G. Houdek ${ }^{9}$, R. A. García ${ }^{10}$, and W. J. Chaplin ${ }^{5}$ \\ 1 Univ. Paris-Sud, Institut d'Astrophysique Spatiale, UMR 8617, CNRS, Bâtiment 121, 91405 Orsay Cedex, France \\ e-mail: Thierry.Appourchaux@ias.u-psud.fr \\ 2 Tata Institute of Fundamental Research, Homi Bhabha Road, 400005 Mumbai, India \\ 3 Sydney Institute for Astronomy (SIfA), School of Physics, University of Sydney, New South Wales 2006, Australia \\ ${ }^{4}$ Department of Astronomy, The University of Tokyo, Tokyo 113-033, Japan \\ 5 School of Physics and Astronomy, University of Birmingham, Edgbaston, Birmingham B15 2TT, UK \\ ${ }^{6}$ Instituto de Astrofísica de Canarias, 38205 La Laguna, Tenerife, Spain \\ 7 Universidad de La Laguna, Dpto. de Astrofísica, 38206 La Laguna, Tenerife, Spain \\ ${ }^{8}$ LESIA, Observatoire de Paris, CNRS UMR 8109, UPMC, Université Denis Diderot, 5 place Jules Janssen, 92195 Meudon Cedex, \\ France \\ 9 Stellar Astrophysics Centre, Department of Physics and Astronomy, Aarhus University, 8000 Aarhus C, Denmark \\ 10 Laboratoire AIM, CEA/DSM-CNRS-Université Paris Diderot, IRFU/SAp, Centre de Saclay, 91191 Gif-sur-Yvette Cedex, \\ France
}

A\&A 566, A20 (2014), DOI: 10.1051/0004-6361/201323317

Key words. stars: interiors - asteroseismology - methods: data analysis - errata, addenda

In Appourchaux et al. (2014), the correct Eq. (1) should have been written with a minus sign in front of the second bracket as

$\ln (\Gamma)=\left(\alpha \ln \left(v / v_{\max }\right)+\ln \Gamma_{\alpha}\right)-\left(\frac{\ln \Delta \Gamma_{\mathrm{dip}}}{1+\left(\frac{2 \ln \left(v / v_{\mathrm{dip}}\right)}{\ln \left(W_{\mathrm{dip}} / v_{\max }\right)}\right)^{2}}\right)$,

where $v$ is the mode frequency, $v_{\max }$ is the frequency of maximum mode height, $\alpha$ is the power law index, $\Gamma_{\alpha}$ is the factor of the power law, $\Delta \Gamma_{\text {dip }}$ is the depth of the dip, $W_{\text {dip }}$ is the width of the dip and $v_{\text {dip }}$ is the frequency of the dip. The fits in Appourchaux et al. (2014) were indeed performed with the minus sign.

Another error was also found in the original Table 3 of Appourchaux et al. (2014) pretending to give the parameters of Eq. (1). As a matter of fact, in that Table 3, the parameter $W_{\text {dip }}$ as reported is related to the full width at half maximum (FWHM) calculated in frequency space, and in addition wrongly computed as it should have been about 1.5 times larger. Using Eq. (1) given above, we can deduce the proper relation between FWHM dip and $W_{\text {dip }}$ as

$F W H M_{\text {dip }}=v_{\text {dip }}\left|\sqrt{\frac{W_{\text {dip }}}{v_{\text {max }}}}-\sqrt{\frac{\nu_{\text {max }}}{W_{\text {dip }}}}\right|$.
In Table 1, we give the parameters that were not affected by the mistake supplemented by the value of $v_{\text {dip }}$ which was not given in Appourchaux et al. (2014). In Table 2, we give the parameter $W_{\text {dip }}$ that was not properly given in the Table 3 of Appourchaux et al. (2014), together with the corrected FWHM $_{\text {dip }}$, a value which was not correct in Table 3 of Appourchaux et al. (2014) and was wrongly named. We also note that from the expression of Eq. (1), there are two solutions for $W_{\text {dip }}$ since we have

$\ln \left(W_{\mathrm{dip}}^{a} / \nu_{\max }\right)=-\ln \left(W_{\mathrm{dip}}^{b} / \nu_{\max }\right)$

where $W_{\text {dip }}^{a}$ is the solution greater than $v_{\max }$, while $W_{\text {dip }}^{b}$ is the solution smaller than $v_{\max }$. Here the solution chosen for $W_{\text {dip }}$ is the former solution.

These corrections do not affect the conclusions reached for the dependence of these parameters upon the effective temperature and $v_{\max }$.

Acknowledgements. We are grateful to Mikkel N. Lund for having found a discrepancy leading to the main author to find the source of the mistake.

\section{References}

Appourchaux, T., Benomar, O., Gruberbauer, M., et al. 2012, A\&A, 537, A134 Appourchaux, T., Antia, H. M., Benomar, O., et al. 2014, A\&A, 566, A20 Benomar, O., Bedding, T. R., Mosser, B., et al. 2013, ApJ, 767, 158 

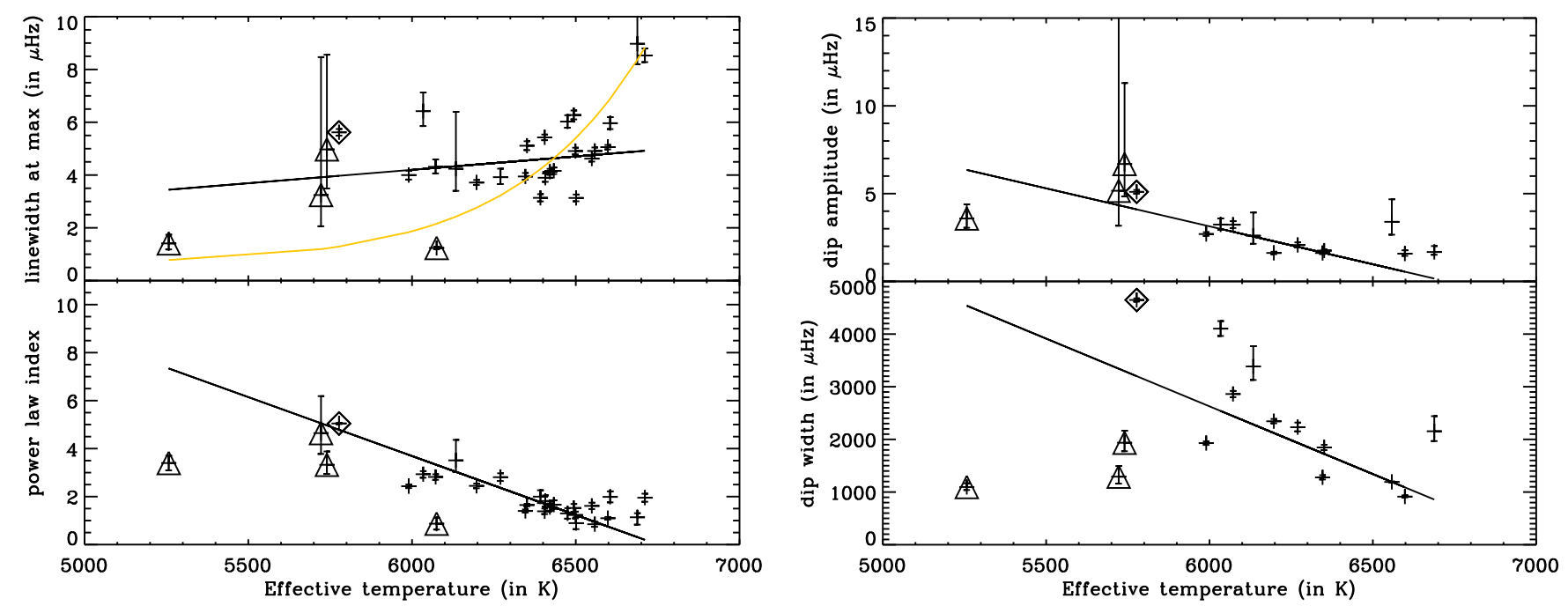

Fig. 6. Parameters of Eq. (1) as a function of the effective temperature for all 28 stars, for the power law dependence (left) and for the Lorentzian fit (right). The median value together with the credible intervals at 33\% and 66\% were derived from a Monte-Carlo simulation of the fit. The orange line shows the temperature dependence of the linewidth at the frequency of maximum mode height as derived by Appourchaux et al. (2012). The open diamond is the result of the fit for the solar data of the LOI. The open triangles are the results of the fit for the sub-giant stars of Benomar et al. (2013). The solid lines show a linear fit of the parameters with respect to the effective temperature. The Lorentzian parameters for stars for which the Lorentzian fit is not significant are not plotted.
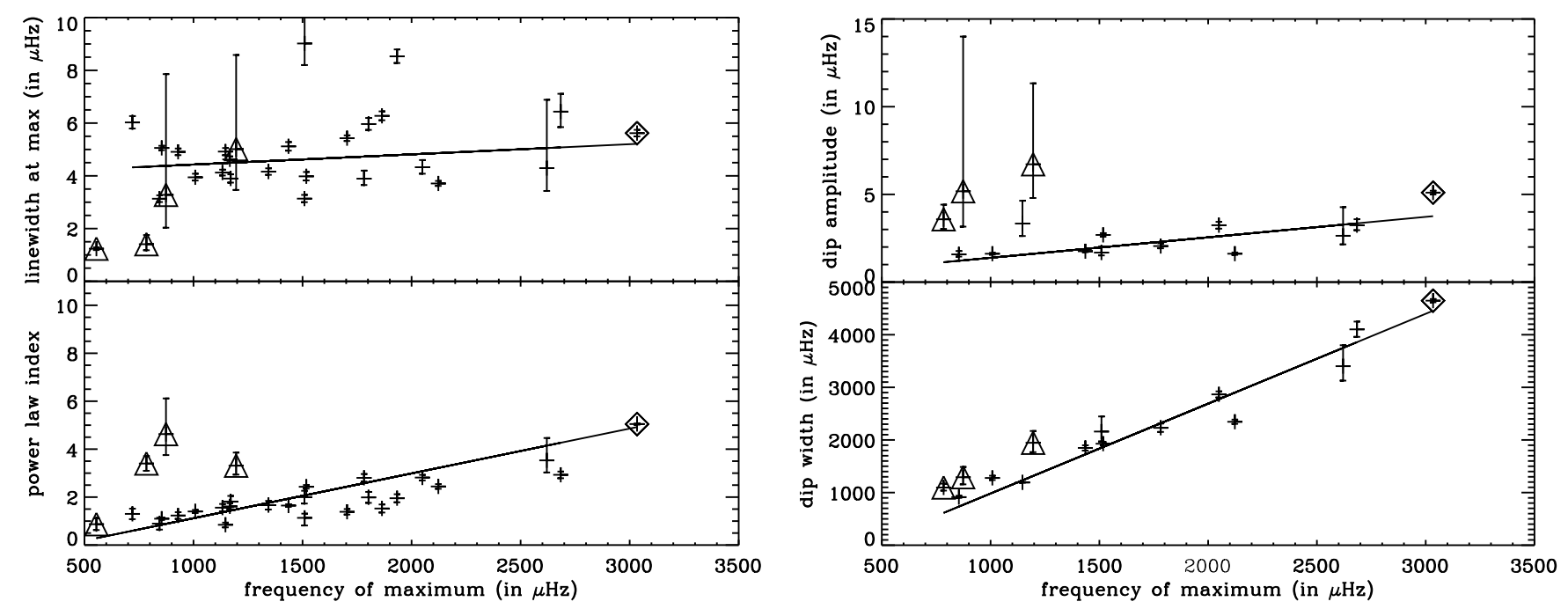

Fig. 7. Parameters of Eq. (1) as a function of the frequency of maximum mode height for all 28 stars, for the power law dependence (left) and for the Lorentzian fit (right). The median value together with the credible intervals of $33 \%$ and $66 \%$ were derived from a Monte-Carlo simulation of the fit. The open diamond is the result of the fit for the solar LOI data. The open triangles are the result of the fit for the sub-giant stars of Benomar et al. (2013). The error bars are derived from a Monte-Carlo simulation using credible intervals of 33\% and 66\%. The solid lines show a linear fit of the parameters with respect to the frequency. The Lorentzian parameters for stars for which the Lorentzian fit is not significant are not plotted. 
T. Appourchaux et al.: Oscillation mode linewidths and heights of 23 main-sequence stars observed by Kepler

Table 1. Linewidth parameters as per Eq. (1).

\begin{tabular}{|c|c|c|c|c|c|c|c|c|}
\hline KIC & $\alpha$ & Uncertainty & $\begin{array}{c}\Gamma_{\alpha} \\
\text { (in } \mu \mathrm{Hz}) \\
\end{array}$ & $\begin{array}{c}\text { Uncertainty } \\
\text { (in } \mu \mathrm{Hz} \text { ) }\end{array}$ & $\begin{array}{c}v_{\mathrm{dip}} \\
\text { (in } \mu \mathrm{Hz} \text { ) }\end{array}$ & $\begin{array}{c}\text { Uncertainty } \\
\text { (in } \mu \mathrm{Hz} \text { ) }\end{array}$ & $\begin{array}{c}\Delta \Gamma_{\text {dip }} \\
\text { (in } \mu \mathrm{Hz} \text { ) }\end{array}$ & $\begin{array}{l}\text { Uncertainty } \\
\text { (in } \mu \mathrm{Hz} \text { ) }\end{array}$ \\
\hline 1435467 & 1.66 & $+0.18 /-0.18$ & 4.16 & $+0.12 /-0.12$ & - & - & - & - \\
\hline 2837475 & 1.13 & $+0.17 /-0.31$ & 9.01 & $+1.63 /-0.80$ & 1509 & $+32 . /-44$ & 1.68 & $+0.36 /-0.16$ \\
\hline 3424541 & 1.30 & $+0.21 /-0.21$ & 6.03 & $+0.23 /-0.23$ & - & - & - & - \\
\hline 3733735 & 1.95 & $+0.16 /-0.16$ & 8.53 & $+0.26 /-0.25$ & - & - & - & - \\
\hline 6116048 & 2.82 & $+0.11 /-0.11$ & 4.32 & $+0.28 /-0.24$ & 2049. & $+11 . /-11$ & 3.23 & $+0.20 /-0.19$ \\
\hline 6508366 & 1.23 & $+0.13 /-0.13$ & 4.91 & $+0.12 /-0.12$ & - & - & - & - \\
\hline 6679371 & 1.10 & $+0.05 /-0.05$ & 5.06 & $+0.09 /-0.09$ & 854. & $10 . /-5$. & 1.57 & $+0.19 /-0.12$ \\
\hline 7103006 & 1.56 & $+0.15 /-0.15$ & 4.12 & $+0.11 /-0.11$ & - & - & - & - \\
\hline 7206837 & 2.00 & $+0.26 /-0.26$ & 3.14 & $+0.14 /-0.13$ & - & - & - & - \\
\hline 8379927 & 2.93 & $+0.13 /-0.14$ & 6.45 & $+0.69 /-0.58$ & 2683. & $+18 . /-19$ & 3.25 & $+0.35 /-0.29$ \\
\hline 8694723 & 1.65 & $+0.05 /-0.05$ & 5.11 & $+0.16 /-0.15$ & 1435. & $+7 . /-7$ & 1.76 & $+0.05 /-0.05$ \\
\hline 9139151 & 3.53 & $+0.87 /-0.51$ & 4.31 & $+2.42 /-0.89$ & 2620. & $+57 . /-37$ & 2.64 & $+1.53 /-0.49$ \\
\hline 9139163 & 1.39 & $+0.11 /-0.11$ & 5.43 & $+0.10 /-0.10$ & - & - & - & - \\
\hline 9206432 & 1.52 & $+0.16 /-0.16$ & 6.28 & $+0.17 /-0.16$ & - & - & - & - \\
\hline 9812850 & 1.81 & $+0.23 /-0.23$ & 3.90 & $+0.16 /-0.15$ & - & - & - & - \\
\hline 10162436 & 1.41 & $+0.06 /-0.06$ & 3.95 & $+0.13 /-0.12$ & 1008. & $+7 . /-7$. & 1.62 & $+0.05 /-0.05$ \\
\hline 10355856 & 0.85 & $+0.09 /-0.10$ & 4.91 & $+0.13 /-0.13$ & 1146. & $+3 . /-3$ & 3.36 & $+1.31 /-0.73$ \\
\hline 10454113 & 2.45 & $+0.09 /-0.09$ & 3.71 & $+0.10 /-0.10$ & 2123. & $+12 . /-12$ & 1.62 & $+0.07 /-0.07$ \\
\hline 10909629 & 0.90 & $+0.24 /-0.24$ & 3.13 & $+0.12 /-0.12$ & - & - & - & - \\
\hline 11081729 & 1.99 & $+0.22 /-0.22$ & 5.96 & $+0.23 /-0.22$ & - & - & - & - \\
\hline 12009504 & 2.81 & $+0.16 /-0.15$ & 3.91 & $+0.30 /-0.25$ & 1781. & $+14 . /-14$ & 2.05 & $+0.15 /-0.13$ \\
\hline 12258514 & 2.43 & $+0.07 /-0.07$ & 3.99 & $+0.16 /-0.16$ & 1517. & $+5 . /-5$ & 2.69 & $+0.10 /-0.10$ \\
\hline 12317678 & 1.61 & $+0.13 /-0.13$ & 4.62 & $+0.11 /-0.11$ & - & - & - & - \\
\hline 6442183 & 3.31 & $+0.55 /-0.36$ & 5.00 & $+3.40 /-1.57$ & 1190. & $+19 . /-13$ & 6.72 & $+4.41 /-1.91$ \\
\hline 11026764 & 4.73 & $+1.57 /-0.92$ & 3.40 & $+5.60 /-1.28$ & 941. & $+31 . /-22$ & 5.44 & $+11.0 /-2.20$ \\
\hline 12508433 & 3.40 & $+0.29 /-0.29$ & 1.41 & $+0.34 /-0.22$ & 808. & $+8 . /-9$ & 3.60 & $+0.84 /-0.54$ \\
\hline 11771760 & 0.87 & $+0.24 /-0.24$ & 1.24 & $+0.06 /-0.05$ & - & - & - & - \\
\hline Sun & 4.97 & $+0.03 /-0.03$ & 4.65 & $+0.11 /-0.11$ & 3083. & $+3 . /-4$. & 4.66 & $+0.10 /-0.10$ \\
\hline
\end{tabular}

Notes. The first column provide the KIC numbers. The second and third columns provide the power law index and the $66-\%$ credible error. The fourth and fifth columns provides the linewidth factor and the 66- $\%$ credible error. The sixth and seventh columns provides the frequency location of the dip and the $66-\%$ credible error. The eighth and ninth columns provides the depth of the linewidth dip and the 66-\% credible error.

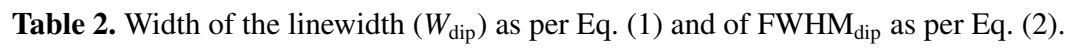

\begin{tabular}{rcccc}
\hline \hline KIC & $\begin{array}{c}W_{\text {dip }} \\
\text { (in } \mu \mathrm{Hz})\end{array}$ & $\begin{array}{c}\text { Uncertainty } \\
\text { (in } \mu \mathrm{Hz})\end{array}$ & $\begin{array}{c}\text { FWHM }_{\text {dip }} \\
\text { (in } \mu \mathrm{Hz})\end{array}$ & $\begin{array}{c}\text { Uncertainty } \\
\text { (in } \mu \mathrm{Hz})\end{array}$ \\
\hline 1435467 & - & - & - & - \\
2837475 & 2154. & $+286 . /-186$ & 537. & $+185 . /-142$. \\
3424541 & - & - & - & - \\
3733735 & - & - & - & - \\
6116048 & 2862. & $+58 . /-60$. & 688. & $+40 . /-44$. \\
6508366 & - & - & - & - \\
6679371 & 913. & $25 . /-15$. & 58. & $+23 . /-14$. \\
7103006 & - & - & - & - \\
7206837 & - & - & - & - \\
8379927 & 4102. & $+144 /-139$. & 1152. & $+99 . /-97$. \\
8694723 & 1846. & $+50 . /-50$. & 364. & $+39 . /-39$. \\
9139151 & 3385. & $+386 . /-257$. & 673. & $+286 . /-208$. \\
9139163 & - & - & - & - \\
9206432 & - & - & - & - \\
9812850 & - & - & - & - \\
10162436 & 1278. & $+42 . /-39$. & 240. & $+33 . /-32$. \\
10355856 & 1192. & $+8 . /-6$. & 44. & $+8 . /-6$. \\
10454113 & 2344. & $+44 . /-38$. & 215. & $+39 . /-37$. \\
10909629 & - & - & - & - \\
11081729 & - & - & - & - \\
12009504 & 2230. & $+88 . /-77$. & 403. & $+66 . /-63$. \\
12258514 & 1929. & $+33 . /-34$. & 365. & $+27 . /-27$. \\
12317678 & - & - & - & - \\
\hline 6442183 & 1936. & $+229 . /-161$. & 582. & $+139 . /-106$. \\
11026764 & 1291. & $+203 . /-131$. & 351. & $+127 . /-97$. \\
12508433 & 1095. & $+71 . /-57$. & 264. & $+51 . /-45$. \\
11771760 & - & - & - & - \\
\hline Sun & 4646. & $+30 . /-31$. & 1303. & $+20 . /-21$. \\
\hline
\end{tabular}

Notes. The first column provide the KIC numbers. The second and third columns provide the width parameter of the dip and the $66-\%$ credible error. The fourth and fifth columns provides the FWHM of the dip and the 66-\% credible error. 